\title{
Le roman en écho. Quand Vincent Almendros dialogue avec Maupassant
}

\author{
Olivier Hambursin \\ Université Saint-Louis - Bruxelles
}

«Le vent se lève, il faut tenter de vivre ». C'est sur ce vers célèbre du Cimetière marin que s'ouvre Un été (2015) de Vincent Almendros. S'il laisse deviner d'emblée la possibilité d'un questionnement sur l'existence, un contexte maritime, l'idée de mouvement, de changement, voire de tempête, il pourrait aussi - on connaît la légende selon laquelle Valéry s'est vu arracher un poème qu'il ne considérait pas comme achevé signifier indirectement au lecteur que ce roman fait écho à une

${ }^{1}$ Désormais $(U E)$ dans cet article. 
autre fiction avec laquelle il dialogue et qu'il vient explorer, déployer, continuer, faire résonner.

C'est la piste que nous proposons de suivre en tentant de montrer que ce court roman, paru aux éditions de Minuit en 2015, peut être lu comme une forme de récriture de Pierre et $\mathrm{Jean}^{2}$ (Maupassant, 1987 [1888]), ce que la critique n'a, semblet-il, pas encore relevé à ce jour.

Nous allons donc essayer d'établir, dans un premier temps, en quoi Un été présente un très intéressant cas contemporain de récriture hypertextuelle, notamment dans son jeu sur la fidélité / infidélité à l'égard du texte de Maupassant, pour dégager ensuite, dans un second temps, quelques fonctions de ce « dialogue » entre deux romans.

\section{Un faisceau de preuves}

\section{ou la fidélité à l'égard de la fiction première}

Affirmer qu'un roman contemporain peut constituer la récriture d'un texte antérieur nécessite, dans le cas où cette relation hypertextuelle n'est pas explicite, d'écarter, autant que possible, le côté aléatoire et potentiellement personnel de l'interprétation du lecteur, "le caractère variable et souvent subjectif de la réception intertextuelle» (Samoyault, 2001, p.67). Contrairement à l'intertextualité au sens large, la récriture, telle que la définit Gignoux (2006) et telle que nous allons ici la concevoir, peut être pensée comme "obligatoire »: «non dans le sens où elle est forcément perçue mais dans le sens où, même si elle n'est pas affichée, une fois démontrée, elle est incontestable et sans

\footnotetext{
${ }^{2}$ Désormais $(P)$ dans cet article.
} 
appel » (Gignoux, 2006, p. 6). Il nous semble donc important, tout d'abord, de mettre au jour ce «faisceau de preuves verbales, reposant toutes sur la répétition, touchant aussi bien les signifiants que les signifiés » (Gignoux, 2006, p. 6) et de repérer «[1]es circulations de sens, [l]es transports de thèmes et de figures » (Samoyault, 2001, p. 90).

Les prénoms des protagonistes constituent un premier niveau de confirmation et peut-être le point décisif susceptible d'amorcer ensuite, en retour, une lecture hypertextuelle.

Dans Un été, le narrateur, seul personnage dont on ne connaît pas d'emblée le prénom, se rend à Naples avec sa compagne, Lone, et embarque sur le bateau de son frère, Jean, qui vit, lui, avec Jeanne. Très vite, on apprend que Jeanne a, auparavant, partagé la vie du narrateur. Profitant d'une absence momentanée de Jean et de Lone, les anciens amants vont se retrouver et s'aimer. C'est au cours de cette scène d'amour que le prénom du narrateur apparaît pour la seule et unique fois dans le roman :

Comme s'il fallait faire vite, ou bien simplement parce que la cabine était étroite, elle s'effondra un peu plus sur moi, laissant aller ses lèvres chaudes sur ma poitrine, respirant ma peau avec un empressement sauvage, cherchant à son tour à retrouver mon odeur. Arrête, dis-je. Elle ne m'écoutait plus. Elle se mit à répéter elle aussi mon prénom. Je n'avais pas du tout envie de l'entendre. J'avais envie de m'oublier. Pierre, insista-telle. Tais-toi, dis-je, ne dis rien. (UE, p. 60-61)

Les prénoms, Pierre et Jean, dans le travail d'identification de l'hypotexte ${ }^{3}$, constituent ici un élément de

3 Ces prénoms permettent bien entendu, comme le rappelle Samoyault, d'« inscrire des noms ou des caractères qui renvoient explicitement à la 
confirmation essentiel, mais, paradoxalement, un indice fragile et ténu également: le lecteur pressé peut ne pas y prêter attention et donc ne plus y être du tout confronté par la suite.

Mais si l'hypotexte se dessine à partir de cette scène centrale, il est confirmé, nous semble-t-il, par quantité d'éléments.

À commencer par l'intrigue, puisqu'on vient de le voir, Un été, comme Pierre et Jean, met en scène deux frères ${ }^{4}$ autour d'une question d'adultère et de liaison extraconjugale (Pierre et Jeanne chez Almendros, la mère de Pierre et de Jean avec Léon Maréchal chez Maupassant), mais aussi deux frères, rivaux, gravitant autour d'une même femme (Jeanne dans Un été, Madame Rosémilly dans Pierre et Jean). De même, les deux romans se terminent par l'isolement, l'exclusion de Pierre: engagement à bord d'un paquebot de croisière comme médecin de bord chez Maupassant et solitude finale du narrateur, chez Almendros, qui, lors du dernier chapitre, sans Lone (elle l'a quitté), retrouve Jean et Jeanne dans leur appartement de Montreuil.

Dans les deux cas également, l'intrigue se noue dans une sorte de huis clos, souvent perçu comme étouffant, qu'il s'agisse de la petite cabine du bateau chez Almendros (« réduit à ciel ouvert », $U E$, p. 40) ou de la maison familiale dans laquelle, une fois l'adultère de Madame Roland suspecté puis avéré, l'atmosphère devient délétère :

littérature antérieure », mais aussi d'«imposer un système de référence autonome » (Samoyault, 2001, p. 74).

${ }^{4}$ Par ailleurs assez proches d'un roman à l'autre puisque, par exemple, dans l'œuvre de Maupassant, Jean est un "grand garçon blond, très barbu » $(P J$, p. 718) et, dans Un été, arbore une « barbe mousseuse » (UE, p. 11). 
Cette maison, la maison de son père l'écrasait. Il sentait peser le toit sur sa tête et les murs l'étouffer. Et comme il avait très soif, il alluma sa bougie afin d'aller boire un verre d'eau fraîche au filtre de la cuisine. (PJ, p. 770-771)

En bas, l'air était irrespirable. [...] Je me sentais de plus en plus nauséeux. (UE, p. 27)

J'avais besoin d'eau comme il arrive d'avoir besoin d'air. J'avais laissé Jeanne s'endormir puis je m'étais levé, j'étais sorti et j'avais regardé l'île de Capri. (UE, p. 62)

En outre, même si les intrigues se déroulent apparemment en des lieux très différents - la Normandie et Le Havre dans l'un, l'Italie (Naples, Capri) dans l'autre -, les deux romans présentent de nombreux points communs en la matière. Ils font de la mer ${ }^{5}$ un élément omniprésent et offrent, par exemple, chacun une "partie de pêche » - l'une à pied dans Pierre et Jean (PJ, p. 787-796), l'autre depuis le bateau, dans Un été (UE, p. 65-68), toutes deux en lien avec des éléments décisifs de l'intrigue: chez Maupassant, Jean demande Madame Rosémilly en mariage au cours de cette partie de pêche et, chez Almendros, cette scène suit directement la brève "liaison" entre Pierre et Jeanne et voit le narrateur s'inquiéter d'avoir oublié sa casquette dans la cabine de son frère.

Mais la mer n'est pas le seul point commun entre l'Italie et la Normandie : non seulement les deux intrigues se déroulent en été ${ }^{6}$, et sous la chaleur, mais en outre, l'Italie est

\footnotetext{
${ }^{5}$ Mer qui est aussi l'objet d'une attention toute particulière de la part de Maupassant (1993 [1886, 1890] et 1993 [1888]), lors de voyages, notamment en Italie.

${ }^{6}$ Coïncidence (ou connivence...) supplémentaire, Pierre et Jean, connu pour sa célèbre « préface », est également présenté comme un roman écrit d'une seule traite par Maupassant, durant... un été : «le roman fut écrit rapidement, en l'espace de trois mois, durant l'été 1887 » (Louis Forestier, dans Maupassant (1987 [1888]), p. 1499).
} 
explicitement mentionnée dans Pierre et Jean. Lorsque Pierre, au chapitre quatre, commence à suspecter la liaison de sa mère et à comprendre donc pourquoi seul son frère Jean va hériter de Léon Maréchal, il se retrouve désespéré sur les quais et se prend à rêver de tout quitter pour... l'Italie et, plus précisément, Naples, ville qui constitue le point de départ de la croisière chez Almendros :

Derrière lui, la voix du veilleur, voix enrouée de vieux capitaine en retraite, criait :

«Le nom du navire? » Et dans le brouillard la voix du pilote debout sur le pont, enrouée aussi, répondit : «Santa-Lucia. - Le pays? - Italie. - Le port? - Naples. » Et Pierre devant ses yeux troublés crut apercevoir le panache de feu du Vésuve tandis qu'au pied du volcan, des lucioles voltigeaient dans les bosquets d'orangers de Sorrente ou de Castellamare! Que de fois il avait rêvé de ces noms familiers, comme s'il en connaissait les paysages! Oh! s'il avait pu partir, tout de suite, n'importe où, et ne jamais revenir, ne jamais écrire, ne jamais laisser savoir ce qu'il était devenu! (PJ, p. 768-769)

Enfin, dans les deux romans, les lieux dans lesquels évoluent les personnages (la Normandie et l'Italie) sont tous les deux liés à Paris et à sa région : c'est à Paris que la mère de Pierre et Jean a rencontré Monsieur Maréchal ; c'est à Paris que la famille Roland a vécu avant de venir s'installer au Havre et, chez Almendros, c'est près de Paris également que se déroule le dernier chapitre, au cours duquel le narrateur retrouve Jean et Jeanne après leur croisière (UE, p. 93-95).

Au-delà de l'intrigue et de son cadre géographique, plusieurs thématiques, plusieurs motifs se retrouvent également d'un roman à l'autre et tissent là encore des liens étroits entre les deux fictions. Retenons-en deux. 
L'orage, tout d'abord. Le vers du Cimetière marin placé en exergue à $U n$ été - « Le vent se lève, il faut tenter de vivre » (UE, p. 7) - peut laisser deviner au lecteur qu'il sera, entre autres, question de changement, d'évolution, voire d'une tempête, ce que le roman confirme : non seulement les quatre protagonistes se trouvent rapidement emportés dans une tempête sentimentale et amoureuse d'envergure, mais en outre, une fois que le narrateur et Jeanne se sont étreints, le temps, météorologique, change et l'orage qui menace finit par s'abattre sur le bateau amarré dans le port d'Agropoli (UE, p. 82). On trouve une situation du même type dans Pierre et Jean : lorsque Pierre, parti réfléchir sur le petit bateau familial, se voit contraint de rentrer car le temps change ${ }^{7}$, mais aussi, quelques pages plus loin, lorsque Pierre convoque un «orage» pour juger, de façon métaphorique, l'absence supposée de remords de sa mère :

Est-ce que toutes les femmes, toutes, n'ont pas cette faculté d'oubli prodigieuse qui leur fait reconnaître à peine, après quelques années passées, l'homme à qui elles ont donné leur bouche et tout leur corps à baiser? Le baiser frappe comme la foudre, l'amour passe comme un orage, puis la vie, de nouveau, se calme comme le ciel, et recommence ainsi qu'avant. Se souvient-on d'un nuage? (PJ, p. 770)

Un été présente, par ailleurs, un étonnant (et passionnant) jeu de chapeaux, et ce, dès les premières pages : Jean arbore « une sorte de large panama dont la calotte était entourée d'un ruban noir » (UE, p. 12) et le narrateur, Pierre, craignant la chaleur, se voit prêter par son frère une casquette

\footnotetext{
7 Cf. « Le matelot dit tout à coup : «V'là d'la brume, m'sieu Pierre, faut rentrer. » Il leva les yeux et aperçut vers le nord une ombre grise, profonde et légère, noyant le ciel et couvrant la mer, accourant vers eux, comme un nuage tombé d'en haut » (PJ, p. 759).
} 
(UE, p. 17-18). Au fil de leur cabotage, les deux couvre-chefs vont être l'objet de nombreuses attentions. Le narrateur, après sa liaison avec Jeanne, va chercher sa casquette, puis, se rendant compte qu'il l'a oubliée sur le lit de Jeanne et de son frère, faire tout pour la récupérer discrètement avant de la retrouver posée sur son lit sans savoir qui l'y a mise (UE, p. 81). Le panama, lui, va passer de la tête de Jean à celle de Lone (UE, p. 64), précisément au moment où ils gagnent la terre ferme pour consulter un médecin (et donc laisser le narrateur et Jeanne seuls), ce qui, à plusieurs reprises, va « déconcerter »le narrateur au point de lui donner «envie de le lui ôter» (UE, p.68). Lone le garde pourtant, puis, après un premier avertissement (UE, p. 84), finit par le perdre puisque le vent l'emporte vers la mer, à la fin de leur voyage (avant-dernier chapitre : $U E$, p. 92).

Ce qui pourrait n'être, en apparence, qu'un simple thème récurrent au sein d'un roman, symbolisant les alliances, les séparations, les changements, voire la trace d'une attention stylistique contemporaine dans la façon d'observer, d'ausculter les objets et les matières, prend pourtant un sens tout différent lorsque l'on considère l'hypotexte. Les chapeaux y sont en effet aussi nombreux et très semblables: Madame Rosémilly - la future épouse de Jean - est affublée d'« un immense chapeau de jardinier, en paille jaune, aux bords démesurés » (PJ, p. 788), et Pierre, embarquant à la fin sur La Lorraine pour New York, porte une «casquette galonnée» (PJ, p. 832); mais surtout, lorsque Pierre essaie de comprendre qui était l'amant de sa mère, comment et dans quelles circonstances ils se sont rencontrés, son père raconte, sans jamais penser qu'il confirme les soupçons de Pierre, une anecdote qui éclaire, dans Un été, ces mouvements de chapeaux : 
Tiens, justement, le matin de la naissance de Jean, c'est lui qui est allé chercher le médecin! Il avait déjeuné chez nous quand ta mère s'est trouvée souffrante. Nous avons compris tout de suite de quoi il s'agissait, et il est parti en courant. Dans sa hâte il a pris mon chapeau au lieu du sien. Je me rappelle cela parce que nous en avons beaucoup ri, plus tard. Il est même probable qu'il s'est souvenu de ce détail au moment de mourir. (PJ, p. 734)

On le voit à travers ces différents éléments - prénoms, intrigue, mer, chapeaux - les liens entre les deux romans sont solides et attestent vraisemblablement qu'Un été peut être lu comme une fiction "seconde», inscrite dans la lignée d'une première, ou, pour reprendre les termes de Genette, comme un cas typique de pratique hypertextuelle et, plus précisément, de «transformation sérieuse » ou de transposition (Genette, 1982, p. 291)

\section{L'écart du double ou l'infidélité de l'hypertexte}

Si la fidélité d'une œuvre seconde par rapport à une fiction première atteste une possible relation hypertextuelle, c'est l'habile travail mis en place pour la dissimuler mais aussi la marge d'infidélité - dans ce qu'elle déplace, prolonge, explore à partir de cette fiction - qui lui donnent son sens, révèlent tout son intérêt. En effet, «l'énonciation réitérante fait toujours subir au texte d'accueil des transformations, envisageables en termes d'altération ou d'augmentation, du contenu comme de la forme » (Samoyault, 2001, p. 74-75).

Un été présente, dans cette perspective, une trame tout à fait personnelle à partir de celle qui structure Pierre et Jean, qui peut donc être perçue comme une forme de « réactivation du sens » (Samoyault, 2001, p.73) de l'hypotexte. L'adultère, 
thème central, est ici non plus celui des parents, comme chez Maupassant, mais le sujet qui va opposer directement deux frères. Par ailleurs, et c'est tout le suspense ménagé par Almendros, l'infidélité se double ici d'une "perversité », d'une machination, d'un calcul absents chez Maupassant. Le dénouement du récit sonne en effet comme un coup de tonnerre ${ }^{8}$, car le narrateur comprend un élément décisif, lorsqu'il vient retrouver son frère et Jeanne, à Montreuil : si Jeanne est venue le rejoindre, si Jeanne et lui se sont aimés durant quelques heures, à bord, c'est qu'elle voulait un enfant, un enfant que Jean ne pouvait lui donner :

[...] je repensais à cet après-midi-là où j'avais cru faire l'amour avec ma Jeanne à moi, mais tout s'éclaircissait à présent, je revoyais sa Jeanne à lui assise dans l'église, son dos religieusement voûté, le regard baissé et les mains jointes, je la revoyais et je sus soudain ce qu'elle réclamait, quelle prière elle faisait, car tout le monde savait que mon frère, à moins d'un miracle, ne pouvait pas avoir d'enfant. (UE, p. 95)

La courte liaison amoureuse du narrateur et de Jeanne se double donc ici d'une sombre manœuvre, dont Jean ne semble pas être exclu. Mais cette différence, majeure, par rapport au texte de Maupassant, paraît d'autant plus intéressante que le narrateur, venant d'apprendre la nouvelle et saisissant donc qu'il a été trompé (si l'on ose le terme dans les présentes circonstances), ne peut que, ironiquement, déclarer : "C'est super, dis-je » (UE, p. 95 : ce sont les derniers mots du roman). Cette phrase résonne alors étrangement avec une des dernières phrases de Pierre et Jean, prononcée cette fois par Roland, qui, lui, n'a rien compris - ni aux troubles de sa famille, ni à la

\footnotetext{
${ }^{8}$ La première phrase du roman, jouant sur l'ambiguïté de l'expression (« rêve d'enfant»), était pourtant explicite et annonçait cette chute : «Disons que pour mon frère, naviguer était un rêve d'enfant » (UE, p. 9).
} 
liaison de sa femme qui en est à l'origine, ni même deviné le futur mariage de son propre fils (Jean) - et affirme : «Très bien, très bien. C'est parfait. Moi je l'approuve absolument» (PJ, p. 833). La bêtise, l'ignorance béate du mari bourgeois chez Maupassant se trouve donc transmuée pour laisser place à l'antiphrase, à l'ironie, au constat désabusé du narrateur chez Almendros.

L'intrigue n'est pas seule à présenter d'importantes divergences par rapport à la fiction première. Le traitement des personnages s'avère par exemple lui aussi très différent.

Alors que le lecteur peut, chez Maupassant, très vite se faire une idée complète des protagonistes de l'histoire - il connaît leur profession, leur passé à Paris, les rivalités qui les opposent, leur situation financière, leur cadre de vie, leurs aspirations, bref, il peut disposer d'un portrait complet et précis - il est, chez Almendros, confronté à un personnage assez typique de certains romans contemporains, notamment parus chez Minuit, à savoir un narrateur dont on ne sait quasiment rien du passé, du métier qu'il exerce, de ses relations, de ses études, de ses ambitions, etc. On songe, bien entendu, à une série de romans regroupés sous l'étiquette (pratique mais forcément incomplète ou imparfaite) d'écriture "blanche", « impassible », qui, à défaut de composer une école, présentent tout de même « une parenté de manière, tant dans la forme que dans les contenus romanesques » (Tadié et Cerquiglini, 2012, p. 393). Cette parenté consiste, entre autres, à mettre en scène des narrateurs (et des personnages) qui ne sont plus des « héros », dont le passé est souvent inconnu, sans avenir, pris dans un présent qu'ils ne parviennent pas à comprendre, dans 
lequel ils peinent à s'exprimer, se contentant d'observer et d'accepter les choses telles qu'elles sont, etc.

Bonne nuit? demanda-t-elle. C'était une question, oui. Une question qui n'en était pas une. Elle m'interrogeait. C'était assez flou.

Bonne nuit, dis-je.

Elle remonta sur le bateau. [...]

Bonne nuit, dit-elle pour la seconde fois. Ce n'était plus une question. Elle descendit.

Attends, lui dis-je.

Quoi?

Non, rien. (UE, p. 50-51)

Pourquoi tu ne m'as pas dit? me demanda-t-elle. Je restai silencieux. Pourquoi tu ne m'as pas dit que tu avais aimé Jeanne?

De là où nous étions, la mer paraissait infinie.

C'est Jean qui te l'a dit?

Qu'est-ce que ça change?

Je ne sais pas, dis-je.

Je vais partir, dit-elle.

Où ça?

Chez moi, dit-elle.

À Paris?

Non, dit-elle, chez moi, et d'une main elle retint le chapeau qui manqua de s'envoler. (UE, p. 84)

Par rapport à la fiction telle qu'elle est développée par exemple chez Maupassant, on observe donc un net renversement ou, pour reprendre les mots de Tadié et Cerquiglini, « un double mouvement inverse » :

Ce qui est traditionnellement considéré comme romanesque (une rupture, un départ, une rencontre, ...) est traité comme un événement sans importance; à l'inverse, de petits événements sont «montés en épingle » par un narrateur qui se pose mille questions. (Tadié, Cerquiglini, 2012, p. 395-396) 
Le départ de Lone, que nous venons de citer, illustre parfaitement ce mouvement : une rupture majeure s'esquisse et se trouve exprimée en quelques lignes de dialogue, sans le moindre commentaire, alors que - exemple de "petits événements » longuement déployés, pris parmi de nombreux autres - le narrateur est capable de consacrer une dizaine de lignes à la description de Jeanne... écalant un œuf (UE, p. 28).

Si Un été, en la matière, trace sa voie loin de Pierre et Jean, on doit bien entendu constater qu'il propose en outre d'importantes transformations narratives et stylistiques. En termes d'infidélité par rapport à l'hypotexte, on retiendra donc aussi une différence majeure - et évidente - d'écriture.

C'est notamment dans l'habile travail sur le non-dit que les textes divergent. Tout, dans Pierre et Jean, est fait pour opposer, dès le départ, les deux frères (portraits, attitudes, réflexions) et ainsi, avant même la révélation de l'infidélité de leur mère, exposer la rivalité qui les oppose :

Les deux frères, en deux fauteuils pareils, les jambes croisées de la même façon, à droite et à gauche du guéridon central, regardaient fixement devant eux, en des attitudes semblables, pleines d'expressions différentes. ( $P J$, p. 733)

Et la remarque faite par la fille de brasserie que Jean était blond et lui brun, qu'ils ne se ressemblaient ni de figure, ni de démarche, ni de tournure, ni d'intelligence, frapperait maintenant tous les yeux et tous les esprits. (PJ, p. 750)

Tout est donc explicite, commenté et développé: la colère, le questionnement, les doutes, les révélations et, bien sûr, le sentiment de jalousie. Le terme est d'ailleurs récurrent dans Pierre et Jean et apparaît quasiment dans chaque chapitre et en tout cas plus de dix fois dans le roman: 
Mais une vague jalousie, une de ces jalousies dormantes qui grandissent presque invisibles entre frères ou entre sœurs jusqu'à la maturité et qui éclatent à l'occasion d'un mariage ou d'un bonheur tombant sur l'un, les tenait en éveil dans une fraternelle et inoffensive inimitié. Certes ils s'aimaient, mais ils s'épiaient. (PJ, p. 719)

En toute autre occasion il n'aurait certes pas compris, pas même supposé possibles des insinuations de cette nature sur sa pauvre mère, si bonne, si simple, si digne. Mais il avait l'âme troublée par ce levain de jalousie qui fermentait en lui. $(P J$, p. 757)

Dans Un été, en revanche, le terme est quasiment inexistant. La jalousie, les rivalités, les tensions sont semblables (c'est la présence, la trace de l'hypotexte), elles sont palpables, mais elles ne sont jamais explicitement formulées, le narrateur se contentant d'observer, de noter, de constater les déplacements, les regards, les objets, les êtres qui l'entourent :

J'avais les bras chargés lorsque Jeanne, en passant devant moi, me souhaita une bonne nuit.

Jean lui demanda de l'attendre, se rinça, cracha, et ensemble, ils regagnèrent leur cabine en refermant la porte derrière eux.

Je regardai cette porte.

Je les imaginais derrière.

Je me tournai vers Lone, qui s'était endormie, une main dans son livre refermé. (UE, p. 45)

Discrètement, j'avançai le long du passavant en m'accrochant aux haubans. Je gagnai l'avant du bateau où le capot de la cabine était relevé. En me penchant, je vis, en contrebas, Jean et Jeanne qui dormaient. Juste à côté de mon frère, ma casquette était posée, en évidence sur le drap. (UE, p. 76)

On peut aussi, pour mettre au jour ces « écarts », prendre deux scènes relativement semblables - elles évoquent toutes deux la violence, le besoin de vengeance éprouvés par Pierre - 
et constater les différences de traitement. Dans Pierre et Jean, de nouveau, le propos est explicite :

Et Pierre se leva, frémissant d'une telle fureur qu'il eût voulu tuer quelqu'un! Son bras tendu, sa main grande ouverte avaient envie de frapper, de meurtrir, de broyer, d'étrangler! Qui? tout le monde, son père, son frère, le mort, sa mère! ( $P J$, p. 768)

À aucun moment, dans Un été, le narrateur ne formule ainsi colère, vengeance ou envie de meurtre. Pourtant, une scène laisse entrevoir une pulsion agressive du même ordre. Le narrateur s'est levé avant les autres et s'apprête à se baigner quand il découvre que la mer est ponctuée de méduses : «Une autre tache, flottant à quelques centimètres de la première, apparut alors, puis une autre, puis encore une autre, et lorsque je compris qu'une dizaine de méduses encerclaient le bateau, je remontai rapidement» (UE, p. 77). Lorsque Jean le rejoint un peu plus tard, il l'encourage à plonger puis feint de ne pas avoir vu la menace :

Il se frotta le visage et ébouriffa ses cheveux, s'étira et bomba le torse en se contorsionnant. Puis il me demanda si je m'étais déjà baigné.

Oui, dis-je.

Il se gratta le thorax et grimaça. C'était la première fois qu'il se levait avant Jeanne.

Il attendit un instant, immobile devant l'échelle.

Allez, saute.

Tu as vu? dit-il soudain. Quoi? Là, me montra-t-il. Je m'étais approché en feignant de découvrir ce qu'il désignait, les cloques qui se promenaient à la surface de l'eau. Son visage s'était fermé. (UE, p. 78)

On le perçoit aisément au travers des quelques extraits exploités ici : Un été peut certes être lu comme une seconde fiction, faisant écho à Pierre et Jean, mais ce texte compose aussi (et peut-être surtout) un roman à part entière, dans lequel un 
écrivain crée et explore un univers, un style qui lui sont propres et lui ont valu, par ailleurs, d'obtenir le Prix Françoise Sagan en 2015.

La phrase d'Almendros est d'apparence simple, mais les descriptions des paysages, des objets, des gestes sont précises, minutieuses, détaillées, à même de faire sentir (plutôt que d'expliquer) le drame qui se joue, l'affleurement des émotions, des sensations, dans un habile travail sur le non-dit : « rien n'est appuyé. Tout est suggéré », comme le résume très justement Jérôme Garcin (2015), mais dans une langue travaillée qui confirme certainement «la présence d'un auteur particulièrement doué » (Fillon, 2015, p. 47).

\section{Du bon usage et des fonctions de la récriture}

Nous aimerions, en guise de conclusion, considérer sous un autre angle le rapport entre ces deux fictions, notamment en envisageant la question des fonctions de la récriture. Si l'on part du principe que toute œuvre est forcément - à des degrés très divers - écho, développement, récriture d'une ou plusieurs œuvres antérieures, qu'apportent des cas «avérés» de récriture tels qu'Un été? Quels peuvent être les intérêts, les rôles du travail ici esquissé? Comment utiliser, exploiter ces mécanismes de la récriture?

On ne peut pas nier un des intérêts majeurs issu de l'analyse du lien qui unit une fiction à une autre, à savoir la dimension de plaisir. Un cas de récriture comme le propose Un été présente en effet une dimension ludique, entre autres parce qu'en partie contraignante. Nous ne préjugerons pas du point de vue de l'auteur en la matière, mais en ce qui concerne le 
lecteur, une fois l'hypotexte suspecté, il devient indéniablement « un complice actif, devant décrypter la parole oblique qui lui est proposée » (Lugrin, 2006, p. 409) et il se voit presque naturellement obligé de relire le roman d'Almendros en ce sens, de se (re)plonger ${ }^{9}$ dans celui de Maupassant et donc d'explorer un « entre-deux».

Car là réside la particularité du plaisir dispensé par la lecture de ces écritures doubles : une telle lecture est elle-même, à la fois, lecture redoublée et dédoublée. Attentifs aux frictions de l'hypertexte et de son hypotexte, voire au télescopage de leurs diverses implications, les lecteurs sont conduits à lire non seulement tel texte particulier mais, pour le moins, un entredeux : celui que délimite l'intervalle fluctuant inhérent à toute relation d'hypertextualité. (Wagner, 2002, p. 302)

Un entre-deux, un dialogue, susceptible de procurer une certaine émotion - placée sous le signe d'une double rencontre - comme le rappelle Gignoux : «celle de l'auteur qu'il lit, dont la voix lui parvient à travers ses mots, et celle, plus lointaine encore, de l'auteur avec lequel le livre dialogue » $(2006$, p. 3). Le plaisir d'identifier ces points de friction, les parallélismes comme les divergences, relève d'un travail d'enquête minutieux, souvent jouissif, mais toujours réflexif. Jenny parle de «machine perturbante », dans la mesure où «il s'agit de ne pas laisser le sens en repos» (1976, p. 279). En somme, ce faisant, on redécouvre ou l'on fait redécouvrir, dans une perspective pédagogique, le paradoxal mouvement de liberté propre à la lecture et à l'analyse: «la lecture des œuvres

\footnotetext{
9 Le nom du bateau dans Un été nous paraît en ce sens hautement symbolique: Reviens, que note non sans ironie le narrateur lorsqu'il le découvre, fait évidemment allusion au fait que Jeanne va lui demander, ponctuellement, de revenir vers lui, mais invite peut-être aussi le lecteur à se retourner, à explorer l'hypotexte, bref, à revenir à Maupassant.
} 
littéraires nous oblige à un exercice de fidélité et de respect dans la liberté de l'interprétation » (Eco, 2002, p. 13).

Revenu aux textes, contraint de les ausculter dans les moindres détails, le lecteur découvre une autre fonction intéressante de la récriture: l'approche comparative et analytique des œuvres littéraires qu'elle engendre et, ainsi, une approche du phénomène littéraire. La récriture, donc l'intertextualité au sens large, semble composer un instrument très efficace pour « engag[er] toute la réflexion sur la nature, les dimensions et la mobilité de l'espace littéraire " (Samoyault, 2001, p. 77). Le travail de relecture, quasiment imposé par ce genre de texte, offre en effet aux enseignants, et notamment à ceux qui peinent parfois à initier leurs étudiants à l'analyse littéraire - quelle qu'elle soit - un indispensable passage de l'activité de lecture à l'approche herméneutique de la littérature: "compte tenu de sa dimension constitutivement relationnelle, l'hypertextualité constitue (entre autres) une incitation à passer graduellement d'une lecture empathique à une lecture stratégique; ou plutôt à concilier ces deux approches » (Wagner, 2002, p. 303). Autrement dit, elle peut permettre de rendre le lecteur "critique», c'est-à-dire, pour reprendre les mots de Samoyault, capable d'identifier les références et allusions, mais surtout de «travaille[r] le sens, en le construisant dans l'entre-deux des textes en présence» (2001, p. 71). Que peut-on apprendre, dès lors, de cette lecture « stratégique », en partie dirigée?

La récriture hypertextuelle, dans la mesure où elle combine une fiction à une autre, constitue un terrain d'enquête idéal pour approcher plusieurs dimensions de la littérature. 
Parmi elles, on pourra retenir la question de l'(in)achèvement des œuvres littéraires et donc de leur relative «ouverture». Étudier les liens étroits qu'Un été tisse avec Pierre et Jean permet de constater à la fois la « clôture » du texte (il peut se suffire à lui-même ; c'est un objet figé, publié, abouti) comme son ouverture (il est toujours susceptible d'être repris, continué, transformé, pastiché, analysé, critiqué, etc.) C'est cette fonction essentielle de l'intertextualité que souligne Rabau lorsqu'elle voit en elle "un moyen d'élargir le texte clos, de penser l'extériorité du texte sans renoncer à sa clôture » (2002, p. 22), rappelant, dans le même mouvement, que l'intertextualité est aussi, pour reprendre le joli titre de Samoyault, une mémoire de la littérature.

Cette prise de conscience en entraîne presque nécessairement une autre, évidence pour les littéraires avertis, moins explicite pour certains étudiants, à savoir que le "principal champ de référence» de la littérature est «la littérature, que les textes interagissent à l'intérieur de ce champ ainsi que dans celui, plus étendu, de l'ensemble des arts » (Samoyault, 2001, p. 55) ${ }^{10}$ - permettant alors de concevoir autrement ces relations intertextuelles, non comme "simple » jeu d'écriture ou pratique d'analyse, mais comme réellement constitutives de la littérature.

Enfin, il nous semble plus aisé d'aborder des concepts comme ceux de lecture, de lecteur, voire d'histoire de la

\footnotetext{
${ }^{10} \mathrm{Ou}$, pour reprendre les termes initiaux de Kristeva, que « tout texte se construit comme une mosaïque de citations, tout texte est absorption et transformation d'un autre texte » $(1969$, p. 85).
} 
lecture $^{11}$, à partir d'une analyse de ces fictions intertextuelles, dans la mesure où elles impliquent davantage le lecteur et s'avèrent donc susceptibles d'amorcer une réflexion en profondeur sur le rôle de la lecture: «il est certain que le concept [...] d'intertextualité » permet « une approche nouvelle et fructueuse du phénomène littéraire et de la lecture » (Picard, 1986, p. 245). Le questionnement relatif à la compétence du lecteur - inhérent à toute réflexion intertextuelle - et suscité par l'approche de cas de récritures tels que ceux proposés par Un été peut ainsi réveiller, développer la compétence du lecteur: «le travail d'intertextualité [...] ne fait pas que présupposer une compétence chez le lecteur (la connaissance d'autres textes); il fait accéder le lecteur à une compétence » (Marguerat, Curtis, 2000, p. 217), une approche critique, une lecture stratégique, qui double finalement celle de l'auteur, tant la récriture, elle aussi, peut être pensée comme lecture : «toute écriture est compte rendu de lecture, jeu avec les possibles livresques, élucubration dans la bibliothèque » (Samoyault, 2001, p. 65).

Bien éloigné d'un «ressassement immobiliste (réexploitation intéressée des recettes des devanciers) », Un été nous semble donc proposer, entre autres, une de ces récritures qui s'avèrent propres à «favorise[r] une mutation novatrice du littéraire » (Wagner, 2002, p. 311) en offrant au lecteur (comme à l'étudiant et au professeur) de nombreux voyages: d'une fiction à l'autre, de l'Italie à la Normandie, d'une forme d'écriture minimaliste au réalisme et au naturalisme, de la

\footnotetext{
${ }^{11}$ Cf. entre autres Picard (1986), Jouve (1993 et 2005) ou encore Dufays et al. (2005).
} 
lecture à l'analyse, de la lecture à l'écriture, etc. Et c'est peutêtre une des fonctions clés de cette dualité fictionnelle - la mise en mouvement de l'esprit critique et analytique - que l'on découvre ici à travers ce passionnant et formateur cabotage littéraire.

\section{Bibliographie}

ALLEN, Graham. (2000), Intertextuality, Londres, Routledge.

ALmEndros, Vincent. (2015), Un été, Paris, Minuit.

BAKHTINE, Mikhaïl. (1978), Esthétique et théorie du roman, Paris, Gallimard, coll. « Tel ».

Barthes, Roland. (1973), Le Plaisir du texte, Paris, Seuil, coll. « Points ».

BÉHAR, Henri. (1981), «La réécriture comme poétique ou le même et l'autre », Romanic Review, vol. 72, n 1, p. 51-65.

BORDAS, Éric. (2002), «Récriture, réécriture», dans Le Dictionnaire du littéraire, Paris, PUF.

-. (2011), L'Analyse littéraire, Paris, Armand Colin.

Compagnon, Antoine. (1979), La Seconde Main ou le travail de la citation, Paris, Seuil.

—. (1998), Le Démon de la théorie, Paris, Seuil, coll. « Points ».

DufaYS, Jean-Louis, Louis GEMEnNE et Dominique LEDUR. (2005), Pour une lecture littéraire, Bruxelles, De Boeck.

ECo, Umberto. (2002), De la littérature, Paris, Grasset.

EnGÉLIBERT, Jean-Paul et Yen-Maï TrAN-GERVAT (dir.). (2008), La Littérature dépliée - Reprise, répétition, réécriture, Rennes, Presses universitaires de Rennes.

FILLON, Alexandre. (2015), « La vie aquatique », Lire, 2015, p. 47. 
GaRCIN, Jérôme. (2015), "Un été" en plein hiver», Le Nouvel Observateur, 12 janvier, <http://bibliobs.nouvelobs.com/lhumeur-de-jerome-garcin/20150112.0BS9749/un-ete-enplein-hiver.html>.

Gauvin, Lise, Cécile Van Den Avenne, Véronique Corinus et Ching SELAO (dir.). (2013), Littératures francophones. Parodies, pastiches, réécritures, Lyon, ENS Éditions.

GenetTe, Gérard. (1982), Palimpsestes. La littérature au second degré, Paris, Seuil, coll. « Points ».

GIGNOUX, Anne-Claire. (2006), « De l'intertextualité à l'écriture », Cahiers de Narratologie, $\mathrm{n}^{\circ} 13, \quad$ p. 2-13, < http://narratologie.revues.org/329>.

—. (2005), Initiation à l'intertextualité, Paris, Ellipses.

Jouve, Vincent. (1993), La Lecture, Paris, Hachette.

—. (2005), L'Expérience de lecture, Paris, L'Improviste.

KiBÉDI-VARGA, Aron. (1990), «Le Récit postmoderne», Littérature, vol. 77, $\mathrm{n}^{\circ}$ 1, p. 3-22.

KristeVA, Julia. (1969), Séméiotikè. Recherche pour une sémanalyse, Paris, Seuil, coll. « Points ».

LUGRIN, Gilles. (2006), Généricité et intertextualité dans le discours publicitaire de presse écrite, Berne, Peter Lang.

MARGUERAT, Daniel et Adrian CURTIS (dir.). (2000), Intertextualités. La Bible en échos, Genève, Labor et Fides.

MAUPASSANT, Guy de. (1993 [1886, 1890]), En Sicile, Bruxelles, Complexe.

—. (1987 [1888]), "Pierre et Jean », dans Romans, édition établie par Louis Forestier, Paris, Gallimard, coll. « Bibliothèque de la Pléiade ».

—. (1993 [1888]), Sur l'eau, Bruxelles, Complexe.

PICARD, Michel. (1986), La Lecture comme jeu, Paris, Minuit. 
PIÉGAY-Gros, Nathalie. (1996), Introduction à l'intertextualité, Paris, Dunod.

Poétique. (1976), Intertextualités, $\mathrm{n}^{\circ} 27$.

RABAU, Sophie (2002), L'Intertextualité, Paris, Flammarion.

RiCARDOU, Jean. (1989), "Pour une théorie de la réécriture ", Poétique, $\mathrm{n}^{\circ} 77$, p. 3-15.

Riffaterre, Michael. (1979), La Production du texte, Paris, Seuil.

—. (1983), Sémiotique de la poésie, Paris, Seuil.

Samoyault, Tiphaine. (2001), L'Intertextualité. Mémoire de la littérature, Paris, Nathan.

SCHLANGER, Judith. (2008), La Mémoire des cuvres, Paris, Verdier.

TAdí, Jean-Yves et Blanche Cerquiglini. (2012), Le Roman d'hier à demain, Paris, Gallimard.

WAGNER, Franck. (2002), « Les hypertextes en questions (notes sur les implications théoriques de l'hypertextualité) ", Etudes littéraires, vol. 34, n 1-2, p. 297-314.

- . (2006), «Intertextualité et théorie », Cahiers de narratologie, vol. 13, <http://narratologie.revues.org/364>.

\section{Résumé}

Un été de Vincent Almendros (Minuit, 2015) peut être lu comme une récriture de Pierre et Jean, ce que la critique n'a, semble-t-il, pas encore relevé. Si l'hypotexte est très habilement dissimulé durant toute la première partie du récit, il devient éclatant au cours d'une scène d'amour adultère, centrale, qui fait apparaître, pour la première et unique fois, le prénom du 
narrateur. L'étude des liens (fidèles et infidèles) que le roman noue avec Pierre et Jean sera l'occasion de réfléchir à la notion de récriture, mais aussi d'esquisser une perspective pédagogique : il semble en effet intéressant d'exploiter le « rôle d'authentique co-énonciateur, dans l'intervalle de l'écriture et de la lecture » (Wagner, 2002) qui est conféré au lecteur dans ce genre de pratique.

\begin{abstract}
Un été by Vincent Almendros (Minuit, 2015) can be read as a rewriting of Pierre et Jean, a point seemingly missed by the reviewer. If the hypotext is very skilfully concealed, throughout the whole of the first part of the story, it flares into view in the course of an adulterous love scene, a pivotal moment, which reveals, for the first and only time, the narrator's first name. Going deeper, studying the connexions (faithful or otherwise) that the novel makes with Pierre et Jean provides an opportunity not only to reflect on the notion of rewriting but also to develop a particular pedagogical perspective: in our view it would be interesting to use the 'authentic co-speaker role arising in the space between the writing and the reading' (Wagner, 2002) that this literary practice bestows upon the reader.
\end{abstract}

